\title{
Crystallization and co-crystallization of CL-20. Influence of some process conditions on product properties
}

\author{
(C) Vladimir N. Popok, ${ }^{*+}$ Nickolay I. Popok, \\ Nickolay V. Bychin, and Svetlana N. Aponyakina \\ Altai Federal Research and Production Center. Socialist St., 1. Bijsk, 659322. Altai Region. Russia. \\ Phone:+7 (3854) 30-19-37.E-mail:vnpopok@mail.ru
}

\begin{abstract}
*Supervising author; ${ }^{+}$Corresponding author
Keywords: CL-20, mixed energy materials, crystallization, crystallization, crystallizer, polymers, sediment, plasticizer, solvent, polymorphic modifications, nitroesters.
\end{abstract}

\begin{abstract}
Crystallization of CL-20 (HNIW) with all the variety of methods faces a number of problems caused by heterogeneous nucleation, polymorphic transformations of the product in the process of crystallization, stability most polymorphic modifications of HNIW under normal conditions, difficulty reproducing granulometric composition and polymorphic purity of the product in laboratory, pilot and industrial facilities. Analysis of literary data shows that important but little researched factors that determine the properties of crystalline product obtained by different methods are: the properties of the crystallizer material; properties of solvents to process the surface of the devices after crystallization; properties of solvents and sediments used in HNIW crystallization. An important element is the analysis of the influence of the type of plasticizers of mixed energy materials (MEM) on the crystallization and co-crystalization processes of HNIW in MEM compositions. It is of some interest to obtain HNIW ultra-dispersing and nanoscale powders in the MEM plasticizer environment, if used as sediments in HNIW crystallization processes.

The article presents the results of experimental studies of influence: the type of material from which the crystallizer is made, the influences of the type of polar solvents used to wash the crystallizer before its subsequent use, influences of the type of MEM plasticizers, as sediments or environments for the creation of the emulsion of HNIW solution in emulsion and sedimentary methods, on crystallization (recrystalization) HNIW; polar polymer-type material for co-crystalization in the polar polymer/HNIW system in thin layers under normal conditions and at cryogenic temperature. The results of the research on the co-crystalization of octogen and HNIW in the environment of inert and nitroester plasticizers of MEM are also presented.

Glass, aluminum, stainless steel, porcelain and fluoroplast crystallizers were used to crystallize HNIW/acetone/o-xylol. Acetone, water, ethanol, $o$-xylol in different combinations or sequences were used to wash crystallizers or to process them further before crystallizing. MEM plasticizers were used as sediments instead of $o$-xylol: transformer oil, dioctyladipineate, propylene glycol, diethylene, triethylene glycol and nitroglycerin. Polar polymer/HNIW casseators used glass, aluminum, stainless steel, fluoroplast and polyethylene to co-crystallize in thin layers at room and cryogenic temperatures. Polyvinilacetate, acetyleulose, nitrocellulose were used as polar polymers. The co-crystalization of HNIW with the octogen was considered in model blends with the above MEM plasticizers.

Studies allow you to choose the conditions for the crystallization and co-crystallization of HNIW with the provision of reproducible characteristics of variability, polymorphic composition, including for the receipt of a high-dispersive product $\varepsilon$-modifications.

The possibility of obtaining a high-dispersal product (crystalline HNIW and its co-crystallizates with MEM components) using SEM plasticizers as sediments or components of mixtures has been confirmed, which is a promising direction alternative technologies for making MEM based on high-dispersal fillers.
\end{abstract}

\section{References}

[1] Xu J., Y. Tian, H. Liu, Y. Shu, J. Sun. Polymorphism in hexanitrohexaazaisowurtzitane crystallized from solution. Journal of Crystal Grows. 2012. Vol.354. P.13-19.

[2] J. Zhang, X. Guo, Q. Jiao, P. Zhang. Phase transitions of e-HNIW in compound systems. AIP ADVANCES. 2016. Vol.6. 10p. 
[3] J. Li., T.B. Brill. Kinetics of Solid Polymorphic Transitions of CL-20. Propellants, Explosives, Pyrotechnics. 2007. Vol.32. No.4. P.326-330.

[4] V.N. Popok. Research on hexanitrohexaazaisowurtzitane/polymer co-crystallizate properties. Butlerov Communications. 2012. Vol.30. No.6. P.132-143. ROI: jbc-02/12-30-6-132

[5] V. Stepanov, R.B. Patel, R. Mudryy, H. Qiu. Investigation of Nitramine-Based Amorphous Energetics. Propellants, Explosives, Pyrotechnics. 2016. Vol.41. P.142-147.

[6] V.N. Popok, N.V. Bichin, P.I. Taronov. Features of thermal decomposition and thermal explosion of compositions with polycrystals of polyethylene glycol, cyclic nitrates and oxidizing agents. Butlerov Communications. 2018. Vol.56. No.12. P.60-70. DOI: $10.37952 / R O I-j b c-01 / 18-56-12-60$

[7] J.H. Urbelis. Altering physical properties of energetic materials and other small molecules through template-directed and co-crystallization methods. Dissertation of Doctor of Philosophy in Chemistry. Georgetown University. Washington. 2014. 337p.

[8] P.V. Nikolaevich, P.N. Ivanovich. Features of some methods of obtaining ultra-and nanodisperse powders hexanitrohexaazaisowurtzitane, explosive properties of powders and compositions on their basis. Butlerov Communications. 2018. Vol.55. No.9. P.58-66. DOI: 10.37952/ROI-jbc-01/18-55-9-58

[9] Zhou Shuiping, Pang A., Tang G. Crystal Transition Behaviors of CL-20 in Polyether Solid Propellants Plasticized by Nitrate Esters with co-Existence of HMX and CL-20. New Journal of Chemistry. 2017. 32p. DOI: 10.1039/C7NJ03309E.

[10] V.N. Popok, and N.V. Bychin. Properties thermoreversible cocrystals with a low temperature of melting on a basis hexanitrohexaazaisowurtzitane. Butlerov Communications. 2014. Vol.37. No.2. P.39-52. ROI: jbc-02/14-37-2-39

[11] D.I.A. Millar, H.E. Maynard-Casely, D.R. Allan, A.S. Cumming, A.R. Lennie, A.J. Mackay, I.D.H. Oswald, C.C. Tang, C.R. Pulham. Crystal engineering of energetic materials: Co-crystals of CL-20. Cryst. Eng. Comm. 2012. Vol.14. P.10. P.3742-3749.

[12] O. Bolton, L.R. Simke, P.F. Pagoria, A.J. Matzger. High Power Explosive with Good Sensitivity: A 2:1Cocrystal of CL-20:HMX. Cryst. Growth Des. 2012. No.12(9). P.4311-4314.

[13] V.N. Popok, N.V. Bichin, and A.A. Averin. Features of interaction of a 2,4-dinitro-2,4-diazapentane and 1,3,5,7-tetranitro-1,3,5,7-tetraazacyclooctane at production and storage the compositions. Butlerov Communications. 2016. Vol.46. No.4. P.1-14. DOI: 10.37952/ROI-jbc-01/16-46-4-1

[14] M.L. Levinthat. Propellant made with cocrystals of cyclotatramethylenetatranitramine and ammonium perchlorate. US Patent №40861 10. 1978.

[15] V.N. Popok, V.I. Desyatykh, and N.I. Popok. Influence of processes of a cocristallization on rheological and mechanical characteristics of mixes on the basis of hexanitrohexaazaisowurtzitane (HNIW). Butlerov Communications. 2013. Vol.35. No.9. P.144-155. ROI: jbc-02/13-35-9-144 